\title{
Semi-Rigid Thoracoscopic Punch Biopsy Using a Hybrid Knife with a High-Pressure Water Jet for the Diagnosis of Pleural Effusions
}

\author{
Yan Yin ${ }^{a}$ Ralf Eberhardt ${ }^{b} \quad$ Xiao-bo Wang ${ }^{a} \quad$ Qiu-yue Wang ${ }^{a} \quad$ Jian Kang ${ }^{a}$ \\ Felix J.F. Herth ${ }^{b}$ Gang Hou ${ }^{a}$ \\ a Department of Respiratory Disease, The First Hospital of China Medical University, Shenyang, China; \\ ${ }^{b}$ Department of Pneumology and Critical Care Medicine, Thoraxklinik University of Heidelberg, \\ Heidelberg, Germany
}

\section{Established Facts}

- Obtaining adequate samples from thickened pleura is the most important limitation of semi-rigid thoracoscopy.

- Mesothelioma and benign fibrothorax (e.g., tuberculous pleurisy or benign asbestos pleural disease) are often challenging to biopsy with a standard flexible forceps.

\section{Novel Insights}

- A hybrid knife with a high-pressure water jet can be used to collect large biopsies of thickened pleura via semi-rigid thoracoscopy.

- This method of obtaining adequate samples from thickened pleura is convenient, effective and safe.

\section{Key Words}

Semi-rigid thoracoscopy · Hybrid knife · Pleural biopsy · Malignant pleural effusion - Tuberculous pleurisy .

Fibrothorax

\section{Abstract}

Semi-rigid thoracoscopy is an important technique in the definitive diagnosis of pleural diseases with high diagnostic sensitivity and specificity. Obtaining adequate samples from thickened pleura is the most important limitation of semi-rigid thoracoscopy with a standard flexible forceps (SFF) compared with rigid thoracoscopy, especially in patients with mesothelioma or benign fibrothorax. Developing a convenient, efficient and safe biopsy technique to obtain sufficient samples from such patients is a key topic in semi-rigid thoracoscopy. The hybrid knife (HK) is an innovative design fusing high-pressure water injection and a conventional diathermic knife that can allow for the safe resection of a larger lesion during gastrointestinal endo-

Yan Yin and Ralf Eberhardt contributed equally to this article.

\section{KARGER}

E-Mail karger@karger.com www.karger.com/res
C 2016 S. Karger AG, Basel

0025-7931/16/0923-0192\$39.50/0
Felix J.F. Herth

Department of Pneumology and Critical Care Medicine Thoraxklinik University of Heidelberg

Amalienstrasse 5, DE-69126 Heidelberg (Germany)

E-Mail felix.herth@med.uni-heidelberg.de 
scopic dissection. Here, we describe 3 patients with unexplained pleural effusion who underwent pleural biopsy using an HK to investigate the potential use of the HK as a new pleural biopsy device in semi-rigid thoracoscopy when pleural lesions are difficult to biopsy using an SFF. The biopsies were obtained successfully by $\mathrm{HK}$, and the diagnosis followed. The sizes of the biopsies collected by HK are larger than those collected by SFF. No complications were observed. Electrocautery biopsy using an HK during semi-rigid thoracoscopy has great potential for diagnosing consistent abnormal pleuras, which are difficult to biopsy with an SFF.

(c) 2016 S. Karger AG, Basel

\section{Introduction}

Semi-rigid thoracoscopy is an important technique in the definitive diagnosis of pleural diseases with high diagnostic sensitivity and specificity [1-3]. Obtaining adequate samples from thickened pleura is the most important limitation of semi-rigid thoracoscopy with a standard flexible forceps (SFF) compared with rigid thoracoscopy, especially in patients with mesothelioma or benign fibrothorax (e.g., tuberculous pleurisy or benign asbestos pleural disease) [4]. The size of the SFF limits biopsies because the SFF lacks mechanical strength to obtain sufficient pleural specimens if fibrotic pleuras were encountered. Since semi-rigid thoracoscopy has advantages including easy maneuverability [5], and less need for analgesia and sedation [6,7], it is used worldwide for the diagnosis of pleural diseases, especially in developing countries. However, determining a convenient, efficient and safe biopsy technique to increase the diagnostic yields of the patients mentioned above is an important goal in semi-rigid thoracoscopy. The hybrid knife (HK) is an innovative design fusing high-pressure water injection and a conventional diathermic knife. It allows for the safe resection of a large lesion during endoscopic dissection (ESD), a popular and effective gastrointestinal endoscopy technique [8]. The physical characteristics of pleural lesions are similar to those of gastroenteric lesions, so we sought to apply HK to pleural biopsy during semi-rigid thoracoscopy. Here, we describe 3 patients with unexplained pleural effusion who underwent pleural biopsy using HK with a high-pressure water jet. To our knowledge, this is the first investigation of the potential use of the HK as a new pleural biopsy device in pleural diseases that are difficult to biopsy with an SFF.

Pleural Biopsy Using a Hybrid Knife via Semi-Rigid Thoracoscopy

\section{Case Reports}

Case 1

A 56-year-old woman admitted to our hospital in April 2016 presented with a cough and shortness of breath of a 2-week duration. The chest computed tomography (CT) scan showed a massive pleural effusion in the right chest cavity. Pleural fluid analysis revealed a yellowish exudate; cultures for bacteria, fungi and mycobacteria were negative. Analysis of pleural effusion and tumor markers are shown in table 1 . The cytological pathology of pleural fluid revealed no evidence of malignancy.

Semi-rigid thoracoscopy (LTF260, Olympus, Tokyo, Japan) was performed under local anesthesia. The incision site was on the mid-axillary line of the right fifth intercostal space. The thoracoscope was introduced into the thoracic cavity via a flexible trocar. Whitish thickened parietal pleural lesions with scattered nodules were observed. We could not obtain specimens from the pleural lesions using an SFF (FB-55CR-1, Olympus) because of their con-

Table 1. Summary of the analysis of pleural effusion and tumor markers

\begin{tabular}{llll}
\hline Items & Case 1 & Case 2 & Case 3 \\
\hline Pleural effusion & & & \\
White blood cell, $\times 10^{6} / 1$ & 1,554 & 950 & 1,201 \\
Mononuclear cell, \% & 48 & 85 & 94 \\
Polymorphonuclear cell, \% & 52 & 15 & 6 \\
Red blood cell, $\times 10^{12} / 1$ & 0.016 & 0.09 & 0.003 \\
Total protein, g/l & 45 & 40 & 35 \\
LDH, U/ml & 246 & 319 & 212 \\
CEA, ng/ml & 118.00 & 80.03 & 0 \\
NSE, ng/ml & 17.92 & 16.04 & 8.72 \\
CA19-9, U/ml & 4.17 & 6.00 & 3.64 \\
CA125, U/ml & 264.5 & 191.3 & 34.56 \\
\hline Serum & & & \\
Total protein, g/l & 55.3 & 63.5 & 48.0 \\
LDH, U/ml & 137 & 165 & 149 \\
CEA, ng/ml & 42.53 & 93.20 & 0.47 \\
NSE, ng/ml & 11.96 & 17.31 & 20.32 \\
CA19-9, U/ml & 2.68 & 3.54 & 6.94 \\
CA125, U/ml & 20.44 & 46.36 & 17.29 \\
\hline
\end{tabular}

$\mathrm{LDH}=$ Lactate dehydrogenase; CEA = carcinoembryonic antigen; NSE = neuron-specific enolase; CA19-9 = carbohydrate antigen 19-9; CA125 = carbohydrate antigen 125.

Fig. 1. a The HK (type T) is a T-shaped tip knife with a water jet in the axial region. $\mathbf{b}$ The HK's access to the whitish pleura with nodules. c The high-pressure water jet $\left(30 \mathrm{~cm} \mathrm{H}_{2} \mathrm{O}\right)$ positioned near the target pleura. $\mathbf{d}$ The HK was used to mark the incision. e Incision of the pleura with the HK. $\mathbf{f}$ The target pleura was incised in $4 \mathrm{~min}$. $\mathbf{g}$ The pleura was collected by cryoprobe. $\mathbf{h}$ The size of the pleural biopsy from case 1. i Comparison of the sizes of biopsies collected by SFF (left) and an HK (right) in case 2.

(For figure see next page.) 

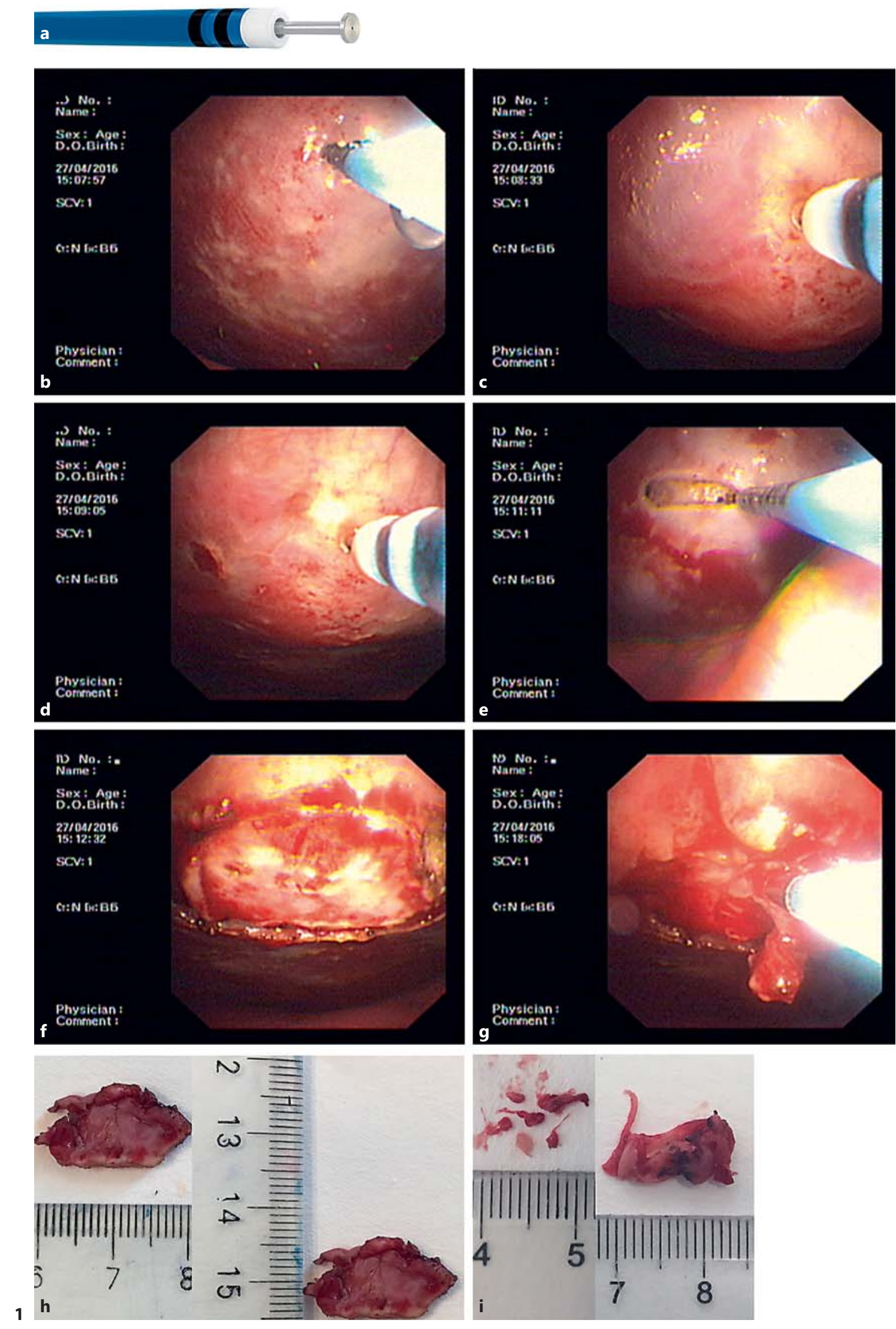
sistency and smooth surface. The VIO 300D generator (ERBE Elektromedizin, Tübingen, Germany) was used as a surgical system. We used a water jet HK (Erbe Hybrid Knife ${ }^{\circledR}$ T-type I-jet, ERBE Elektromedizin) (fig. 1a; online suppl. video 1; for all online suppl. material, see www.karger.com/doi/10.1159/000448556) to perform electrosurgical circumferential cutting and dissection of pleural lesions, and a water jet to inject a solution of $2 \%$ lidocaine and glycerol into the parietal pleura with $30 \mathrm{~cm} \mathrm{H}_{2} \mathrm{O}$ of water jet pressure (fig. 1b, c). After making a pinhole in the margin of elevated pleura (fig. 1d) with the HK, the tip of the HK was inserted into the hole, and a circumferential incision was made (VIO mode ENDO CUT Q, effect3-width1-interval of incision4, Coagulation effect 2 and $30 \mathrm{~W}$ ) (fig. 1e, f). Finally, the incised piece of pleura $(20 \times 10 \mathrm{~mm})$ was collected using a cryoprobe without pleural bleeding or chest pain (fig. $1 \mathrm{~g}, \mathrm{~h}$ ). The procedure time from submucosal injection to the completion of the pleural biopsy was approximately $10 \mathrm{~min}$. The pathological diagnosis was metastatic lung adenocarcinoma, and immunochemical staining showed to be positive for CK7 and TTF-1 and negative for vimentin, P63, CK5/6, and CR.

\section{Case 2}

A 52-year-old man admitted to our hospital in April 2016 presented with cough, hemoptysis and dyspnea upon exertion for 2 months. The chest CT showed a moderate left pleural effusion. Pleural fluid was a serosanguinous exudate. Analysis of pleural effusion and tumor markers are shown in table 1 . The laboratory tests for infections were negative. There was no evidence of malignancy. Semi-rigid thoracoscopy was performed under local anesthesia. Whitish thickened pleura with scattered white-yellow nodules were observed. We used an HK to perform pleural dissection because the pleural lesions were difficult to collect with an SFF. The settings of the instruments were the same as those for case 1 . The incised piece of pleura was $8 \times 12 \mathrm{~mm}$, which was larger than that collected by SFF (fig. 1i). No complications were observed. The procedure time was approximately $10 \mathrm{~min}$. The pathological diagnosis was chronic granuloma with infiltration of lymphocytes, which suggested a diagnosis of tubercular pleurisy. One month after anti-tuberculosis therapy, the pleural effusion was obviously absorbed.

\section{Case 3}

A 29-year-old man admitted to our hospital in April 2016 presented with cough and dyspnea upon exertion for 2 weeks. The chest CT showed a moderate right pleural effusion. The pleural fluid was a yellowish exudate with a predominant increase in the ratio of lymphocytes (94\%). Analysis of pleural effusion and tumor markers are shown in table 1 . The laboratory tests for infections were negative. There was no evidence of malignancy. The patient refused empirical anti-tuberculosis treatment, so we performed semi-rigid thoracoscopy and collected a biopsy with an HK due to the consistency and smooth surface of the pleura, which was similar to that of case 1 . The procedure time was approximately $7 \mathrm{~min}$. The pathological diagnosis was chronic granuloma with infiltration of lymphocytes. Clinical data did not support the possibility of fungal infection, sarcoidosis or rheumatoid arthritis. Therefore, we diagnosed the patient with tuberculosis pleurisy. When we followed up the clinical response 2 months after anti-tuberculosis therapy, the pleural effusion had disappeared.

Pleural Biopsy Using a Hybrid Knife via Semi-Rigid Thoracoscopy

\section{Discussion}

Semi-rigid thoracoscopy is important in the diagnosis of pleural effusions. Compared with rigid thoracoscopy, the main limitation of semi-rigid thoracoscopy is the size of samples obtained by SFF in particular cases, such as malignant or benign fibrothorax. Rigid thoracoscopy can be advantageous because the rigid forceps are larger and more powerful than SFF; however, semi-rigid thoracoscopy also has advantages such as easy maneuverability [5], less need for analgesia and sedation, and less frequent bleeding during the pleural biopsy [6,7]. In order to make up for the limitation of semi-rigid thoracoscopy, new techniques have been introduced to semi-rigid thoracoscopy to improve the quality and size of samples, such as collecting multiple biopsies, taking several 'bites' of the same area [1], cryobiopsy [9], and electrocautery [10]. Sasada et al. [10] first introduced the insulation-tipped (IT) knife, which was used in ESD for pleural biopsy and showed an excellent diagnostic yield. The complementation of novel techniques to improve the quality and size of samples from semi-rigid thoracoscopy is also crucial. $\mathrm{HK}$ is a novel electrosurgical instrument whose function is the fusion of a submucosal injection and electrocautery. A previous study demonstrated that ESD using an $\mathrm{HK}$ is a faster and simpler procedure and requires fewer accessory changes than conventional ESD [11]. Our cases showed that an $\mathrm{HK}$ is also effective in pleuroscopic biopsy when malignant/benign fibrothorax is encountered. Moreover, the procedural time of HK biopsy did not exceed $15 \mathrm{~min}$ in our experience. There are two possible reasons for the short procedural time: one is the highpressure water jet, which made the submucosal injection easier than that using a conventional injection needle; the other is the ability to perform a submucosal injection and incision with one tool, which saves time in switching between the injection needle and electrosurgical knife. Tang et al. [12] revealed that the use of an HK in peroral endoscopic myotomy can shorten the procedural time compared to the conventional method (injection needle and triangular tip knife), which supports our hypothesis. The biopsy size using an $\mathrm{HK}$ is obviously larger than that using SFF, and in cases 1 and 3, SFF was not able to collect appropriately sized biopsies. However, an HK could be used to obtain a sufficient biopsy and a definitive diagnosis. Therefore, medical thoracoscopic biopsy with an HK is a time-saving, convenient and effective technique.

According to a previous study of the IT knife, the potential complications of HK include chest pain and bleeding [10]. The IT knife is a monopolar electrosurgical knife 
that does not combine electrocautery and electrocoagulation; therefore, bleeding is a common complication [13, 14]. However, the HK can perform mixed electrocautery, which may decrease the risk of pleural bleeding.

Our study has several limitations. Our report only contains 3 cases and solely demonstrates the feasibility of the HK in pleural biopsy via semi-rigid thoracoscopy. It should be taken into account that with a higher number of cases the number of complications may increase. Further study is needed to explore the efficacy, safety and the best indications of this technique.
In conclusion, the use of an $\mathrm{HK}$ with a water jet is a potential feasible and convenient biopsy technique for semi-rigid thoracoscopy for the successful diagnosis of thickened pleural lesions. It is notably useful when fibrothorax and/or smooth, thickened lesions are encountered.

\section{Financial Disclosure and Conflicts of Interest}

The authors declare that they have no conflicts of interest.

\section{References}

$\checkmark 1$ Lee P, Mathur PN: Advances in pleural diseases: what is the future for medical thoracoscopy? Curr Opin Pulm Med 2016;22:297308.

2 Hooper C, Lee YC, Maskell N; BTS Pleural Guideline Group: Investigation of a unilateral pleural effusion in adults: British thoracic society pleural disease guideline 2010. Thorax 2010;65(suppl 2):ii4-ii17.

- 3 Wang XJ, Yang Y, Wang Z, Xu LL, Wu YB, Zhang J, Tong ZH, Shi HZ: Efficacy and safety of diagnostic thoracoscopy in undiagnosed pleural effusions. Respiration 2015;90:251255.

4 Yap KH, Phillips MJ, Lee YC: Medical thoracoscopy: rigid thoracoscopy or flexi-rigid pleuroscopy? Curr Opin Pulm Med 2014;20: 358-365.

-5 Ernst A, Hersh CP, Herth F, Thurer R, LoCicero J 3rd, Beamis J, Mathur P: A novel instrument for the evaluation of the pleural space: an experience in 34 patients. Chest 2002;122: 1530-1534.

6 Dhooria S, Singh N, Aggarwal AN, Gupta D, Agarwal R: A randomized trial comparing the diagnostic yield of rigid and semirigid thoracoscopy in undiagnosed pleural effusions. Respir Care 2014;59:756-764.

7 Agarwal R, Aggarwal AN, Gupta D: Diagnostic accuracy and safety of semirigid thoracoscopy in exudative pleural effusions: a metaanalysis. Chest 2013;144:1857-1867.

$\checkmark 8$ De-la-Pena J, Calderon A, Esteban JM, LopezRoses L, Martinez-Ares D, Nogales O, OriveCalzada A, Rodriguez S, Sanchez-Hernandez E, Vila J, Fernandez-Esparrach G: Experimental study of hybrid-knife endoscopic submucosal dissection (ESD) versus standard ESD in a Western country. Rev Esp Enferm Dig 2014;106:98-102.

-9 Thomas R, Karunarathne S, Jennings B, Morey S, Chai SM, Lee YC, Phillips MJ: Pleuroscopic cryoprobe biopsies of the pleura: a feasibility and safety study. Respirology 2015;20: 327-332.

-10 Sasada S, Kawahara K, Kusunoki Y, Okamoto $\mathrm{N}$, Iwasaki T, Suzuki H, Kobayashi M, Hirashima T, Matsui K, Ohta M, Miyazawa T: A new electrocautery pleural biopsy technique using an insulated-tip diathermic knife dur- ing semirigid pleuroscopy. Surg Endosc 2009; 23:1901-1907.

11 Zhou PH, Schumacher B, Yao LQ, Xu MD, Nordmann T, Cai MY, Charton JP, Vieth M, Neuhaus H: Conventional versus Waterjetassisted endoscopic submucosal dissection in early gastric cancer: a randomized controlled trial. Endoscopy 2014;46:836-843.

12 Tang X, Gong W, Deng Z, Zhou J, Ren Y, Zhang Q, Chen Z, Jiang B: Comparison of conventional versus hybrid knife peroral endoscopic myotomy methods for esophageal achalasia: a case-control study. Scand J Gastroenterol 2016;51:494-500.

13 Mori G, Nonaka S, Oda I, Abe S, Suzuki H Yoshinaga S, Nakajima T, Saito Y: Novel strategy of endoscopic submucosal dissection using an insulation-tipped knife for early gastric cancer: near-side approach method. Endosc Int Open 2015;3:E425-E431.

14 Masai K, Sasada S, Izumo T, Taniyama T, Nakamura Y, Chavez C, Sakurai H, Tsuta K, Tsuchida T: Pleuroscopic punch biopsy using insulated-tip diathermic knife-2 for the diagnosis of desmoplastic malignant mesothelioma. J Bronchology Interv Pulmonol 2013;20:345-348. 